\title{
A graph-based modelling methodology for high-pressure networks applied on waterjet machining
}

\author{
S. Niederberger ${ }^{\mathrm{a}, \mathrm{b}, *}$, R. Orjuela ${ }^{\mathrm{a}, * *}$, P. Schleuniger ${ }^{\mathrm{b}}$, R. Anderegg ${ }^{\mathrm{b}}$, M. Basset $^{\mathrm{a}}$ \\ ${ }^{a}$ Université de Haute-Alsace, IRIMAS (EA 7499), 12 rue des Frères Lumire, Mulhouse, 68093 France \\ ${ }^{b}$ University of Applied Sciences Northwestern Switzerland, Institute of Automation, Klosterzelgstrasse 2, Windisch, 5210 Switzerland
}

\begin{abstract}
This paper proposes a graph-based methodology that models high-pressure networks of various topologies. Therefore, a mathematical modelling of a supply network for waterjet machining will be introduced. High-pressure components are assigned to homogeneous segments, each representing a local pressure state as a differential equation. Segments are subsequently interconnected along the fluid flow path as an algebraic equation that allocates a fluid flow to the interconnections, resulting in a lumped parameter model. For this purpose, a graph network description has been used to approximate the spatially distributed high-pressure system. In this way, the proposed methodology offers a flexible modelling to cope with different network topologies. Moreover, a variable fluid compressibility has also been introduced so that a wide operating range can be included. This modelling methodology has been applied to a supply network for waterjet machining. The resulting mathematical model has been verified by measurements from a test bench with a pressure range of 100 to $400 \mathrm{MPa}$. It was shown that a variable fluid compressibility improves the model's accuracy and that modelling errors can be reduced in comparison to other existing methodologies.
\end{abstract}

Keywords: High-pressure network modelling, graph network, lumped parameter model, varying parameter model, waterjet machining

\section{Introduction}

Nowadays, waterjet machining is used in metal, composite, textile, food and many other industries. It is the first choice for contour cutting and surface stripping causing minimal thermal stress and is devoid of chemicals. Besides various pure water applications, abrasive waterjet cutting is often used to increase the material removal rate when machining hard and brittle materials [1]. These applications demand operating pressures in a range of 100 to $400 \mathrm{MPa}$. A pressure of $700 \mathrm{MPa}$ has been reported to further increase the productivity of waterjet cutting [2]. Future research should improve the energy efficiency of entire waterjet facilities. Hence, mathematical modelling is needed to provide valuable numerical simulations that further enhance the performance of waterjet cutting. The present work was initiated by a recently developed, directly driven piston pump. These modular piston pumps allow the realization of a new class of scalable high-pressure networks for waterjet machining. The industry needs a modelling methodology to optimally design future high-pressure networks of next-generation waterjet facilities. This modelling approach is also used to efficiently research distributed control concepts and managing algorithms for various decentralized pump setups. In this respect, different modelling approaches can already be found in the field of waterjet cutting [3] - [6] and in related fields [7] - [10] to model specific high-pressure systems.

In general, the principles of continuity and momentum conservation appear to be the most prevalent approach when modelling high-pressure generation. For waterjet machining, Tremblay et al., 1999 [3] presented a model for intensifier pumps with attenuators. Their experimental studies reveal the effect of bulk modulus, fluid density and other parameters on pressure fluctuations at 153.4, 181.0 and 215.5 MPa. More than a decade later, Fabien et al., 2010 [4]

\footnotetext{
${ }^{*}$ Corresponding author

** Principal corresponding author

Email address: stefan.niederberger@uha.fr (S. Niederberger)
} 
adopted a lumped parameter model for a similar setup. The pressure-dependent fluid density is taken into account. For this purpose, model parameters have been experimentally identified with measurement data at $153.4 \mathrm{MPa}$. Other researchers have modelled high-pressure pumps for diagnostics and condition monitoring, e.g. Modelica is employed by Ferreiit et al., 2015 [5] for a multi-domain simulation of a phased intensifier pump. They also researched piston position and pressure generation to develop a model-based fault detector. However, most authors focus on the modelling of waterjets and the resulting material removal while the high-pressure generation remained ignored. Thus, a generalized material removal model was proposed by Momber, 1995 [6] to study various particle sizes and fluid flow rates for abrasive waterjet cutting.

The study of automotive applications revealed that fuel injection systems for combustion engines are similar to common installations for waterjet machining. A high-pressure pump feeds injectors which are interconnected by pipes. Hountalas et al., 1998 [7] modelled a direct injection engine with an operating pressure of about $50 \mathrm{MPa}$. Reasonable accuracy was realized by introducing a pressure-dependent bulk modulus. Recently, there have been more studies that deal with common rail injection systems. Many of them focus on the injection process, e.g. Wang et al., 2011 [8] who utilize a MATLAB Simulink model to study the injection rate, while others are interested in high-pressure generation and control. For this purpose, Lino et al., 2007 [9] presented a mathematical model for control design and its implementation in MATLAB Simulink. The modelling has a variable bulk modulus. It was experimentally validated for a pressure range of 30 to $90 \mathrm{MPa}$ and augmented with multi-domain simulations in AMESim. Due to control design, a mathematical model was also developed by Wang et al., 2016 [10] and validated using AMESim for a pressure of $150 \mathrm{MPa}$. However, the validation results reveal some discrepancies regarding the transient behavior of pressure generation.

Earlier researchers consistently assume a one-dimensional fluid flow and most of them take into account a pressuredependent fluid density. This fluid density influences the fluid discharge including the pressure steady state. Some authors suggest a variable bulk modulus, e.g. when modelling fuel injection systems. Bulk modulus defines the fluid compressibility and the transient behaviour of pressure generation. The present study combines a variable fluid density with a pressure-dependent bulk modulus. This was done not only to model a wide operating range, as both parameters affect the pressure generation, but also to reduce the number of model coefficients. The coefficients that describe these pressure-dependent parameters can be identified independently of any specific network topology. Accordingly, it is novel to introduce both pressure-dependent parameters into the governing equations and to assign these modified equations to a graph description. In this way, various high-pressure networks can be modelled without revising the initial parameter identification.

The main contribution of this paper is to propose a flexible modelling of high-pressure networks using graphs. Firstly, network components are unitarily assigned to homogeneous segments, resulting in a lumped parameter model in which each segment represents a local pressure state as a differential equation with respect to the principle of momentum conservation. Secondly, these segments are interconnected along the expected fluid flow path, so that a graph network reveals the interconnections, whereas algebraic equations restore the fluid flow between segments with respect to the principle of continuity.

Graphs [11] are often used to represent vehicular traffic or data packet flow. By extending this, Espitia et al., 2017 [12] proposed to model communication networks by means of fluid dynamics. Considering general physical systems, a modelling approach has been provided by van der Schaft, 2017 [13]. Graphs are thereby used in PortHamiltonian systems regarding conservation laws and balance equations. A graph-based approach to model thermal fluid power flow systems with respect to conservation of mass and energy is presented by Koeln et al., 2016 [14] and Pangborn et al., 2017 [15]. Whereas Corbet et al., 2018 [16] map the transient solution of diffusion equation on a graph to describe the fluid flow in petroleum infrastructures. Moreover, Borsche et al., 2016 [17] have investigated conservation laws using graphs to represent spatially distributed physical systems as lumped parameter models. Such models are often applied to thermal systems, e.g. by Ramallo-González et al., 2013 [18]. Numerous studies combine graph theory with dynamic modelling. However, a flexible modelling methodology that considers pressure generation over a wide operating range with reasonable accuracy remains desirable, especially for high-pressure networks.

The presented methodology is considered to be generic because it assumes a generalized description of pressure generation and assigns the corresponding equations by means of graphs. This graph-based approach allows the modelling of common high-pressure components within a uniform framework. The resulting graphs correspond to a 
system of equations which represent modular subsystems. These subsystems are flexibly interconnected to compose the more complex supply networks of entire facilities. In contrast to other approaches, the underlying generalized description considers varying parameters and essential restrictions, enabling the reproduction of the manifold dynamic behaviour of various high-pressure components.

In this paper a graph-based modelling methodology is investigated which numerically simulates supply networks of various topologies with reasonable modelling effort and simulation accuracy. The paper is organized as follows: Section 2 discusses the class of high-pressure network that is addressed with the proposed modelling methodology. A generalized description for homogeneous segments is developed in Section 3 and the expected parameter variations are introduced. A graph-based modelling methodology provides the main contribution when it maps the generalized description to a graph network. Section 4 defines a test bench setup that represents a supply network for waterjet machining and the functional principle of a piston pump is explained. The proposed modelling methodology is used to assign the test bench components to homogeneous segments. Section 5 analyses the pressure-dependent fluid compressibility using experimental data for parameter identification and validates the derived high-pressure network model for a pressure range of 100 to $400 \mathrm{MPa}$. Finally, Section 6 discusses the results and draws conclusion for the future research.

\section{System description}

Waterjet machining installations usually consist of a remote high-pressure pump that directly feeds a work station through a high-pressure pipe as depicted in Figure 1 (a). Common high-pressure pumps pressurize water by means of hydraulically driven intensifiers. Up to now, prototypes of energy-efficient high-pressure pumps have been developed [19]. These prototypes use pistons which are directly driven by independent electro-mechanical actuators. Any number of directly driven pistons can be combined and spatially distributed to simultaneously feed various work stations, as exemplary shown in Figure 1 (b), establishing scalable high-pressure networks of different topologies.

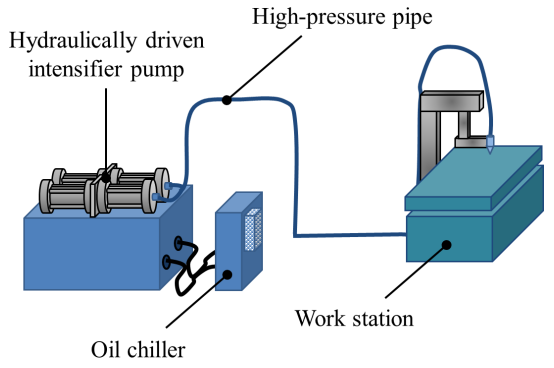

(a) Common high-pressure system for waterjet machining.

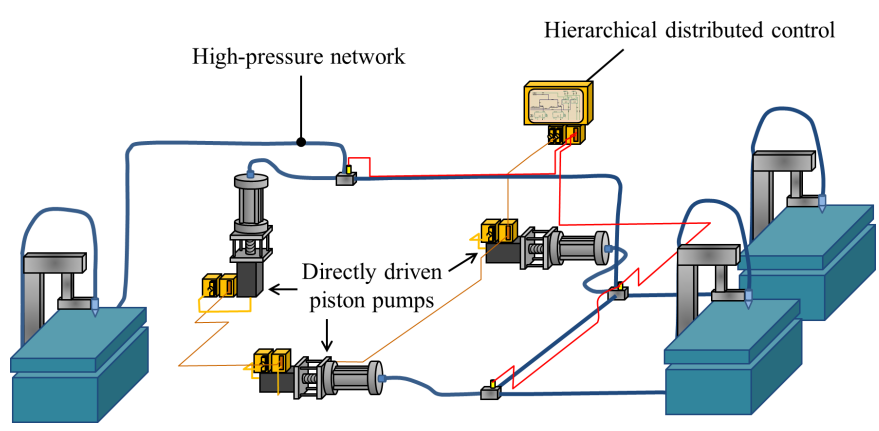

(b) High-pressure network to supply an entire waterjet facility.

Figure 1: Extension of a hydraulically driven high-pressure system (a) to a complex high-pressure network (b) interconnecting three directly driven piston pumps and three waterjet work stations.

This networked approach aims to improve the overall performance, efficiency and reliability of waterjet facilities. However, strong couplings across high-pressure networks require a distributed control of all the interconnected piston pumps. The development of sophisticated control designs needs an appropriate modelling methodology. Such a methodology must also consider non-linearities and varying parameters for a wide pressure range. In addition, it must ensure the flexible modelling of arbitrary network topologies that interconnect any number of fluid flow sources, e.g. piston pumps, and pressure sinks, e.g. work stations.

The proposed modelling methodology is expected to flexibly cope with various network topologies. Scalable high-pressure networks should become straightforward to model. The resulting mathematical model should remain accurate without having to revising parameter identification and model validation. Applying this methodology to a supply network for waterjet machining exemplifies a system with wide operating ranges. Therefore, a test bench was developed to simulate scalable high-pressure networks with various piston pumps which simultaneously operate at different network positions. A detailed system description is given in Section 4 when the test bench setup is introduced. 


\begin{tabular}{|c|c|c|}
\hline \multicolumn{2}{|c|}{ Nomenclature: } & \multirow[b]{2}{*}{$(\mathrm{m} / \mathrm{s})$} \\
\hline$a$ & Fluid speed of sound & \\
\hline$c_{1}, c_{2}$ & Friction loss parameters & $(-),(-)$ \\
\hline$D$ & Cross section diameter of a segment & (m) \\
\hline $\begin{array}{l}f(\cdot), f_{k j}(\cdot) \\
J\end{array}$ & $\begin{array}{l}\text { Flow path switching function of a segment, switching function of branch } k j \\
\text { Set of nodes }\end{array}$ & $(-)$ \\
\hline$i$ & Index of measurement data & $(-)$ \\
\hline j & Index of target node $j$ & $(-)$ \\
\hline$K, K_{0}$ & Fluid bulk modulus, initial fluid bulk modulus & (Pascal) \\
\hline$k$ & Index of original node $k$ & $(-)$ \\
\hline$k j$ & Index of branch $k j$ (from original node $k$ to target node $j$ ) & $(-)$ \\
\hline$L, L_{k}$ & High-pressure component initial length, initial length of segment $k$ & (m) \\
\hline$l, l_{k}$ & Initial length of a segment, initial length of node $k$ & (m) \\
\hline$N$ & Number of nodes in a graph & $(-)$ \\
\hline$p, p_{k}$ & Pressure state of a segment, pressure state of node $k$ & (Pascal) \\
\hline$p_{\text {Sup. }}$ & Water supply pressure & (Pascal) \\
\hline$p_{i}$ & Measured pressure sample $i$ & (Pascal) \\
\hline$Q, Q_{k j}$ & Fluid flow rate of a segment, flow rate of branch $k j$ & $\left(\mathrm{~m}^{3} / \mathrm{s}\right)$ \\
\hline$Q_{E x t .}, Q_{k}$ & Displacement flow rate of a piston, displacement flow rate of node $k$ & $\left(\mathrm{~m}^{3} / \mathrm{s}\right)$ \\
\hline & Reynolds number & $(-)$ \\
\hline$r_{s}$ & Trajectory set point variable (positions, velocities) & $(\mathrm{mm}),(\mathrm{mm} / \mathrm{s})$ \\
\hline$S, S_{k}$ & Cross section of a segment, cross section of node $k$ & $\left(\mathrm{~m}^{2}\right)$ \\
\hline$S_{E x t .}, S_{k j}$ & Cross section of a piston, cross section of branch $k j$ & $\left(\mathrm{~m}^{2}\right)$ \\
\hline$t, t_{0}$ & Time coordinate, time initial condition & (s) \\
\hline$u_{m}$ & Measured control variables (positions, velocities) & $(\mathrm{mm}),(\mathrm{mm} / \mathrm{s})$ \\
\hline$V, V_{k}$ & Fluid volume of a segment, fluid volume of node $k$ & $\left(\mathrm{~m}^{3}\right)$ \\
\hline$V_{i}$ & Measured fluid volume sample $i$ & $\left(\mathrm{~m}^{3}\right)$ \\
\hline$w$ & Fluid flow velocity of a segment & $(\mathrm{m} / \mathrm{s})$ \\
\hline$w_{E x t}, w_{k}$ & Displacement velocity of a piston, displacement flow velocity of node $k$ & $(\mathrm{~m} / \mathrm{s})$ \\
\hline$y_{m}, y_{e}$ & Measured and simulated process variables (pressures) & (Pascal) \\
\hline$x$ & Flow path position coordinate & (m) \\
\hline$\alpha, \alpha_{k j}$ & Discharge coefficient of a segment, discharge coefficient of branch $k j$ & $(-)$ \\
\hline$\Delta p, \Delta p_{k j}$ & Pressure difference of a segment, Pressure difference of node $k$ and $j$ & (Pascal) \\
\hline$\Delta Q, \Delta Q_{k j}$ & Flow rate difference of a segment, total flow rate difference on node $k$ & $\left(\mathrm{~m}^{3} / \mathrm{s}\right)$ \\
\hline$\Delta S_{k j}$ & Cross section difference from node $k$ to branch $k j$ & $\left(\mathrm{~m}^{2}\right)$ \\
\hline$\Delta x$ & Length of a segment & (m) \\
\hline$\kappa_{0}, \kappa_{1}$ & Fluid bulk modulus parameters & (Pascal), (-) \\
\hline$\lambda$ & Friction loss coefficient of a segment & $(-)$ \\
\hline$\rho, \rho_{0}$ & Fluid density, initial fluid density & $\left(\mathrm{kg} / \mathrm{m}^{3}\right)$ \\
\hline $\mathcal{E}, e$ & Set of branches and branch (edge) & \\
\hline $\mathcal{G}$ & Set of graphs & \\
\hline $\mathcal{N}, \mathcal{N}_{k j}$ & Set of networks, high-pressure network & \\
\hline $\mathcal{V}, v$ & Set of nodes and node (vertices) & \\
\hline
\end{tabular}

\section{Modelling methodology}

In this section, a graph-based modelling methodology for high-pressure networks is introduced. For this purpose, the dynamics of a homogeneous segment has been described bearing in mind the principle of continuity and momentum conservation. The resulting equations have been simplified by assuming a stationary fluid flow and in addition, parameter variations have been introduced. A graph description reveals the initial fluid flow path, resulting in a generic network description. 


\subsection{Dynamics of a homogeneous segment}

High-pressure components are evenly divided along their fluid flow path to establish homogeneous segments of constant cross sections $S$ and lengths $\Delta x$, as shown in Figure 2. Each segment holds a finite fluid volume $V=S \Delta x$. A high-pressure component determines the direction of fluid flow, whereby a one-dimensional fluid flow is assumed. The position coordinate $x$ has been introduced to define the fluid flow in a positive direction, e.g. from a piston pump to a cutting head.

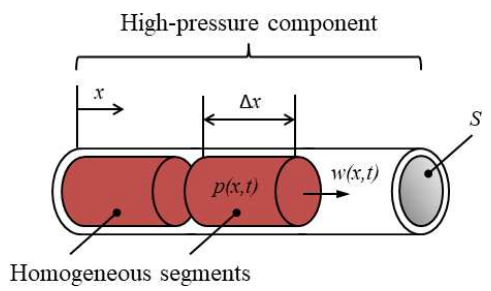

Figure 2: Illustration of a high-pressure component assigned to homogeneous segments of length $\Delta x$ with the pressure state $p(x, t)$ and fluid flow velocity $w(x, t)$. The position coordinate $x$ defines the direction of positive fluid flow.

The governing partial differential equations [20]:

$$
\begin{aligned}
\frac{\partial}{\partial t} p(x, t)+a^{2} \rho \frac{\partial}{\partial x} w(x, t) & =0 \\
\frac{\partial}{\partial t} w(x, t)+\frac{1}{\rho} \frac{\partial}{\partial x} p(x, t) & =-\frac{\lambda}{2 D} w(x, t)|w(x, t)|
\end{aligned}
$$

are applied to describe the dynamics of the homogeneous segments. This approach is common in many studies that model high-pressure generation, e.g. [3], [7], [9] and [21]. The dependent variables $p(x, t)$ and $w(x, t)$ refer to the pressure state and to the fluid flow velocity. Both of them depend on the position coordinate $x$ and time $t$. On the one hand, the principle of continuity (1) indicates that a velocity gradient along the position coordinate causes a pressure variation in time. On the other hand, the momentum conservation (2) dictates that a pressure gradient along the position coordinate will induce a velocity variation in time, which means, the fluid is either accelerated or decelerated.

Since the speed of sound $a$ and density $\rho$ are fluid characteristic constants, the cross section diameter $D$ and friction loss coefficient $\lambda$ are parameters of specific high-pressure components. It is further noted that the resulting fluid flow will cause a friction loss which is sensitive to the flow direction and proportional to the squared velocity.

To obtain ordinary differential equations, the following Taylor series expansion:

$$
f(x+\Delta x)=\sum_{n=0}^{\infty} \frac{\frac{d^{n}}{d x^{n}} f(x)}{n !} \Delta x^{n}=f(x)+\frac{d}{d x} f(x) \Delta x+O(\Delta x)
$$

is used, in which $\Delta x$ denotes the length of a segment. By assuming that the higher-order terms $O(\Delta x)$ become small, the polynomial (3) can be truncated and rearranged to approximate the first order derivatives in $x$. This approximation is introduced in the backward differential formulation:

$$
\frac{\partial}{\partial x} w(x, t) \approx \frac{w(x, t)-w(x-\Delta x, t)}{\Delta x}
$$

for equation (1) and in forward differential formulation:

$$
\frac{\partial}{\partial x} p(x, t) \approx \frac{p(x+\Delta x, t)-p(x, t)}{\Delta x}
$$

for equation (2). The backward formulation (4) refers to the fluid flow difference relative to a previous segment and the forward formulation (5) consequently indicates a pressure difference relative to a subsequent segment. This definition is advantageous for the introduction of the graph, described at the end of this section. 
The fluid flow velocity is substituted by the fluid flow rate according to $w(x, t)=S^{-1} Q(x, t)$ and the notations:

$$
\begin{gathered}
\Delta Q(t):=Q(-\Delta x, t)-Q(t) \\
\Delta p(t):=p(t)-p(\Delta x, t)
\end{gathered}
$$

are defined for $x=0$, hence the ordinary differential equations:

$$
\begin{aligned}
\frac{d}{d t} p(t) & \approx \frac{a^{2} \rho}{S \Delta x} \Delta Q(t) \\
\frac{d}{d t} Q(t) & \approx \frac{S}{\rho \Delta x} \Delta p(t)-\frac{\lambda}{2 D S} Q(t)|Q(t)|
\end{aligned}
$$

are obtained from (1) and (2), describing the dynamics of a homogeneous segment. As an increased flow rate difference $\Delta Q(t)$ induces the pressure $p(t)$, an increased pressure difference $\Delta p(t)$ yields a flow rate $Q(t)$. Consequently, the flow rate difference will decrease until a pressure equilibrium has been reached.

To allocate these differential equations to a lumped parameter model, an explicit formulation must be derived. A stationary fluid flow has therefore been assumed, to solve (9) in its equilibrium:

$$
\frac{d}{d t} Q(t)=0
$$

This results in an algebraic equation that substitutes (9), whereby the lumped parameter model:

$$
\begin{aligned}
\frac{d}{d t} p(t) & \approx \frac{a^{2} \rho}{S \Delta x} \Delta Q(t) \\
Q(t) & \approx S \sqrt{\frac{2}{\rho} \frac{D}{\Delta x \lambda}} \sqrt{|\Delta p(t)|} \operatorname{sign}(\Delta p(t))
\end{aligned}
$$

is obtained. These equations approximate the local pressure state of a homogeneous segment, including its nonlinearity due to interconnections to the neighbouring segments. The signum function is required to maintain the fluid flow direction. The resulting algebraic equation (12) is also known as a discharge equation, see [20] or [22]. It has been used in many studies, e.g. [9], [10] and [23], to describe the fluid flow through system components such as nozzles, etc.

\subsection{Parameter variations}

High-pressure networks have parameter variations which are either related to fluid characteristics or to specific component properties. It is useful to introduce the largest parameter variations so as to address a wide pressure range and to subsequently provide a generalized description of pressure generation. This parameter variations will be discussed below.

Fluid volume. The transient behaviour of pressure generation in (11) depends on the fluid volume:

$$
V(t)=S \Delta x
$$

This fluid volume can vary for specific high-pressure components. In particular, a piston displacement changes the volume of a pumping chamber. This variable fluid volume is expressed as:

$$
V(t)=S l-\int_{t_{0}}^{t_{0}+t} Q_{E x t .}(\tau) d \tau
$$

when considering the initial segment length $l$ and the displacement flow rate:

$$
Q_{\text {Ext. }}(t)=S_{\text {Ext. }} w_{\text {Ext. }}(t)
$$

if a piston with cross section $S_{E x t}$ is displaced with the velocity $w_{E x t}(t)$. The volume variation of (14) and the displacement flow of (15), respectively, are a priori known periodic functions and therefore are considered to be an external system excitation. However, the displacement flow rate of most components is $Q_{E x t}(t)=0 \forall t$. 
Fluid compressibility. The transient behaviour of pressure generation in (11) is also dependent on the bulk modulus $K(p)$, hence the Newton-Laplace formula:

$$
K(p)=a^{2} \rho
$$

was used to assign the speed of sound $a$ and the fluid density $\rho$ to the bulk modulus. Both fluid characteristic parameters are expected to vary according to the operating pressures. Consequently, a linear parametric model of form:

$$
K(p)=\kappa_{0}+\kappa_{1} p
$$

is frequently proposed, see [7] and [9], to describe a pressure-dependent bulk modulus. However [20], [22], as well as other researchers express the bulk modulus as:

$$
K(p)=\frac{d p}{d \rho / \rho}
$$

Combining (17) with (18) while separating the variables and integrating yields:

$$
\int \frac{1}{\kappa_{0}+\kappa_{1} p} d p=\int \frac{1}{\rho} d \rho
$$

and referring to [2] and [4], an explicit solution is derived from (19) and rearranged to represent a pressuredependent fluid density:

$$
\rho(p)=\rho_{0}\left(1+\frac{\kappa_{1} p}{\kappa_{0}}\right)^{1 / \kappa_{1}}
$$

whereby $\rho_{0}$ denotes the initial density.

In this respect, the variable compressibility affects the transient behaviour of (11) as well as the steady state given by the discharge equation (12). Its coefficients $k_{0}$ and $k_{1}$ are experimentally identified for pressure ranges up to $400 \mathrm{MPa}$ as described in Section 5.

Flow resistance. The discharge coefficient:

$$
\alpha=\sqrt{\frac{D}{l \lambda}}
$$

is introduced into (12). It represents a flow resistance that is individually derived for each high-pressure component with respect to the model segmentation. For example, the fluid flow characteristics of a pipe determine the segment's flow resistance, which is shown in Section 4.

Flow path. Eventually, function $f(\cdot)$ is appended to (12) which determines the existence of a fluid flow along a fluid flow path. This function depends either on time $f(t)=[0,1]$ as if an external signal switches an on/off-valve, or it is associated with a dependent variable as if a check-valve restricts the flow direction. The Heaviside function:

$$
f(\Delta p):= \begin{cases}1 & : \Delta p>0 \\ 0 & : \Delta p \leq 0\end{cases}
$$

is proposed to describe the second case as the switching behaviour depends on the pressure difference $\Delta p(t)$ between segments.

These parameter variations are combined with the lumped parameter model. The varying parameters (13) and (17) are considered for the differential equation (11) as well as a possible induced displacement flow rate (15) is added. The varying parameter (20) and the discharge coefficient (21) are considered for the algebraic equation (12) as well as a possible switching law (22) is introduces. This results in the generalized description:

$$
\begin{aligned}
\frac{d}{d t} p(t) & \approx \frac{\kappa_{0}+\kappa_{1} p(t)}{V(t)}\left(\Delta Q(t)+Q_{E x t .}(t)\right) \\
Q(t) & \approx f(\cdot) S \alpha \sqrt{\frac{2}{\rho_{0}}\left(1+\frac{\kappa_{1} p(t)}{\kappa_{0}}\right)^{-1 / \kappa_{1}}} \sqrt{|\Delta p(t)|} \operatorname{sign}(\Delta p(t))
\end{aligned}
$$

for homogeneous segments, whereby each segment represents a local pressure state. A pressure-dependent bulk modulus has been taken into account and a variable density was introduced to permit a wide operating range. 


\subsection{Generic network description}

To reproduce various network topologies, homogeneous segments are interconnected by means of graph theory [11]. The graph $\mathcal{G}=(\mathcal{V}, \mathcal{E})$ consists of branches or edges $\mathcal{E}$ that interconnects nodes or vertices $\mathcal{V}$. Thus, (23) assigns each node $v \in \mathcal{V}$ to a pressure state $v \mapsto p$ such as:

$$
\frac{d}{d t} p(t): \mathcal{V} \rightarrow \mathbb{R}
$$

and (24) maps each branch $e \in \mathcal{E}$ to a corresponding fluid flow $e \mapsto Q$ such as:

$$
Q(t): \mathcal{E} \rightarrow \mathbb{R}
$$

This results in a network description $\mathcal{N}=(\mathcal{G}, d p / d t, Q)$ of homogeneous segments to represent specific high-pressure networks using lumped parameters. Its components are consequently assigned to $J \rightarrow \mathbb{N}$ homogeneous segments. Each segment represents a node $k \in J$ that is interconnected with other nodes $j \in J \backslash k$ as illustrated in Figure 3. As each node represents a pressure state $p_{k}(t)$, every branch is characterized by a flow rate $Q_{k j}(t)$ with positive flow direction from segment $k$ to $j$.

(a) Homogeneous segment
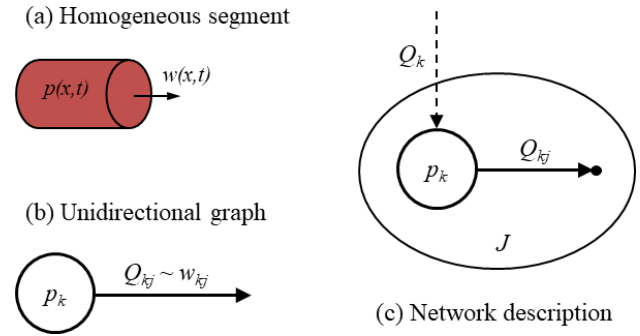

(c) Network description

Figure 3: Representation of segment (a) as a graph (b) and scheme for segment assignment of $k \in J$ nodes in a network (c). Each node $k$ is interconnected to the other nodes $j \in J \backslash k$ by branches $k j$.

Hence, the pressure state of a segment is assigned to a network node:

$$
p(t) \rightarrow p_{k}(t)
$$

and the fluid flow rate between segments is assigned to a network branch:

$$
Q(t) \rightarrow Q_{k j}(t)
$$

The differential scheme (7) becomes:

$$
\Delta p(t) \rightarrow \Delta p_{k j}(t):=p_{k}(t)-p_{j}(t)
$$

that couples interconnected nodes and (6) is substituted by:

$$
\Delta Q(t) \rightarrow \Delta Q_{k j}(t):=\sum_{j=1}^{N} Q_{k j}(t)
$$

to determine $N$ simultaneous interconnections of a node $k$ to any other node $j:=\{1, \ldots, N\}$. A possible displacement flow is assigned to: $Q_{E x t .}(t) \rightarrow Q_{k}(t)$. The sum in (30) conserves the flow rate on every node with respect to the fluid flow direction:

$$
Q_{j k}(t)=-Q_{k j}(t)
$$

Therefore, the output flow rate $Q_{k j}(t)$ from a previous segment yields an input flow rate $Q_{j k}(t)$ to a subsequent segment. With the above introduced assignments (27) to (30), the generic network description of a high-pressure network:

$$
\mathcal{N}_{k j}:= \begin{cases}\frac{d}{d t} p_{k}(t) & =\frac{\kappa_{0}+\kappa_{1} p_{k}(t)}{V_{k}(t)}\left(\sum_{j=1}^{N} Q_{k j}(t)+Q_{k}(t)\right) \\ Q_{k j}(t) & =f_{k j}(\cdot) S_{k j} \alpha_{k j} \sqrt{\frac{2}{\rho_{0}}\left(1+\frac{\kappa_{1} p_{k}(t)}{\kappa_{0}}\right)^{-1 / \kappa_{1}}} \sqrt{\left|\Delta p_{k j}(t)\right|} \operatorname{sign}\left(\Delta p_{k j}(t)\right)\end{cases}
$$


is obtained. Each node $k$ holds a fluid volume $V_{k}(t)$. The function $f_{k j}(\cdot)$ determines the fluid flow along a branch $k j$ which is characterized by the discharge coefficient $\alpha_{k j}$ and the interconnection's cross section $S_{k j}$.

The use of graphs assigns homogeneous segments to the initial fluid flow path. This results in a system of equations considering the network description (32). This system of equations models the generation of pressure for a spatially distributed physical system. The segmentation of high-pressure components is realized according to the following procedure:

1. Identify the high-pressure components which are dedicated to changing the cross section along its fluid flow path.

2. Divide the high-pressure network along the identified components into segments $k$ of lengths $L_{k}$ wherever a cross section changes such as $S_{k} \neq S_{k+1}$.

3. Chose a propagation time $\tau$ which adequately represents the expected dynamics of pressure generation induced by any exogenous fluid flow $Q_{k}$.

4. If $L_{k}>a \tau$, subdivide the initial segments with respect to the fluid's speed of sound $a$ so that $n_{k}$ homogeneous segments of length $l_{k}<a \tau$ are obtained for $n_{k}=\left\lfloor L_{k} / l_{k}\right\rfloor$. Otherwise, $l_{k}=L_{k}$.

5. Simulate the derived network model with the exogenous dynamics on $Q_{k}$ to investigate whether the desired dynamic range of pressure generation has been met.

6. Repeat steps 3 to 5 with varying propagation times to provide a reasonable trade-off between model complexity and accuracy.

As a result, a constant cross section is preserved for each segment. These segments are subdivided, if needed, to satisfy the underlying principles of continuity and momentum conservation. However, it must be mentioned that not every initial segment needs further partitioning into equally spaced homogeneous segments to accomplish the desired dynamic range for simulation.

\section{Application and Implementation}

To validate the proposed modelling methodology, a high-pressure test bench was built that supports various network topologies. In this section, an initial test bench setup is introduced and its functional principle explained. The proposed modelling methodology is then applied to this test bench setup to obtain a high-pressure network model.

\subsection{Test bench setup}

The test bench, as shown in Figure 4, is configurable to represent different waterjet facilities. The present setup includes a directly driven high-pressure pump and a cutting head. The high-pressure pump has two pistons, which are independently positioned by electro-mechanical actuators. Each piston pump represents a source of fluid flow. The cutting head generates a waterjet that is immediately discharged into a container. This represents a pressure sink to the atmosphere. Such a cutting head can be equipped with nozzles of different sizes to reproduce the discharge flow of various work stations. This test bench is flexible enough to simulate different supply network topologies as a desired number of piston pumps can be interconnected with cutting heads by pipe sections of various lengths. In the setup of Figure 4, high-pressure pipes directly interconnect the two piston pumps with a cutting head. For purposes of validation, the pressure was measured after the high-pressure pump as well as before the cutting head. As a result, the coupling of two piston pumps, the pressure generation over a wide operating range and the influence of the varying parameters could be evaluated.

Figure 5 illustrates the analysed high-pressure network. Two pistons are displaced by the piston's velocities $w_{1}$ and $w_{2}$ to generate the displacement flow rates $Q_{1}$ and $Q_{2}$. These cause the pumping chamber pressures $p_{1}$ and $p_{2}$. The outtake ducts of each pressure cylinder contain internal check-valves, which ensure the unidirectional outtake flow rates $Q_{13} \geq 0$ and $Q_{23} \geq 0$ from the pumping chambers to the connector. The outtake ducts reduce the fluid flow cross sections for the first time before it passes the check-valves. A coupling connects the piston pumps to the high-pressure pipes, where the cross section is further reduced, while the outtake flows of both cylinders are merged to the overall flow rate $Q_{34}$. At this position, a pressure transducer has been installed to measure the test bench input pressure $p_{3}$. The test bench is set up with high-pressure pipes that directly feed a cutting head, where a second pressure transducer 


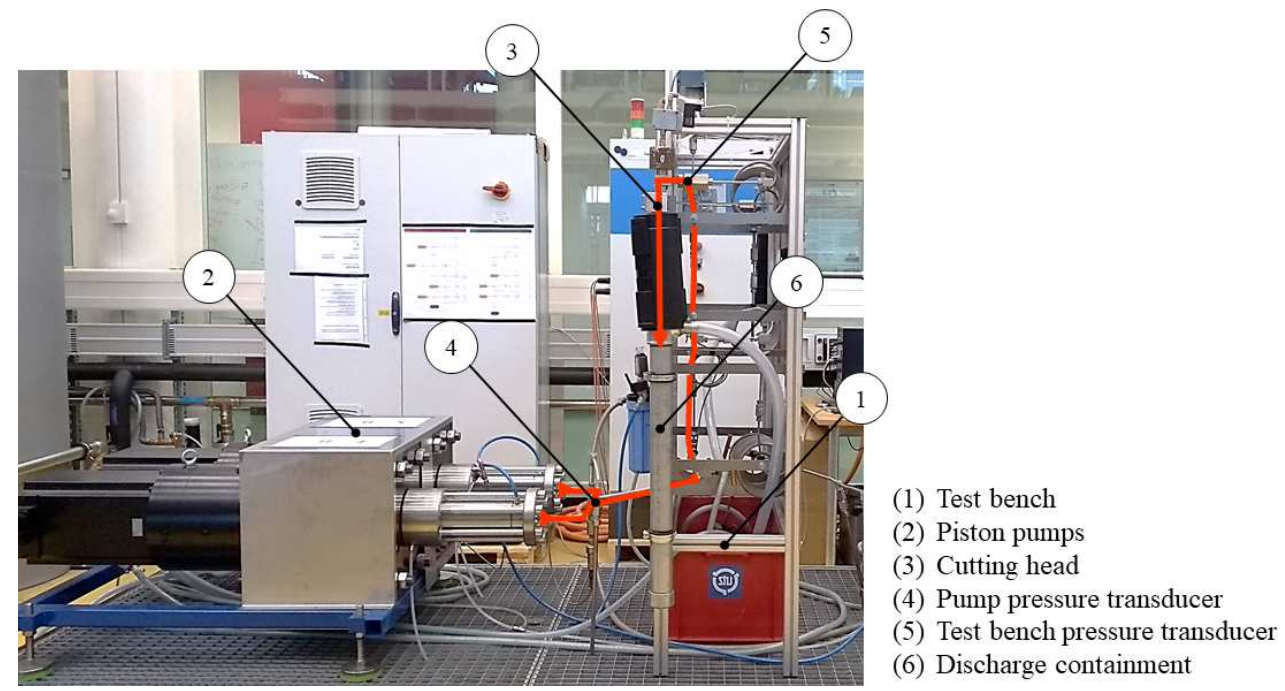

Figure 4: Laboratory with high-pressure test bench configured to interconnect a directly driven high-pressure pump with a cutting head. Highpressure transducers are located at pump outtake and test bench outtake. The generated waterjet is discharged into an safety container.

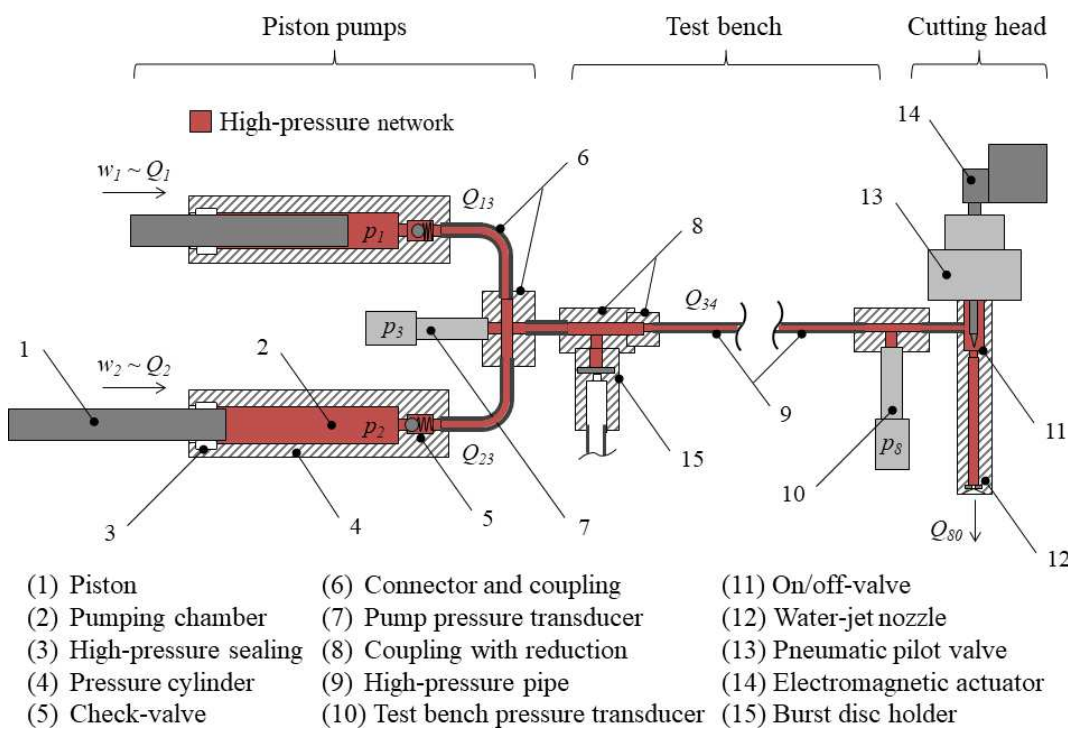

Figure 5: Overview of the investigated high-pressure network as configured on the test bench. Two pistons induce independent displacement flow rates to feed a cutting head which generates a discharge flow.

measures the test bench output pressure $p_{8}$. The cutting head has an on/off-valve and a nozzle. A pneumatic pilot valve switches the on/off-valve. If the on/off-valve is in the open-position, the nozzle will generate a water-jet that results in the discharge flow rate $Q_{80}$. This nozzle reduces the fluid flow cross section one last time. As a piston displacement causes an input flow, the nozzle size defines the output flow based on to the nozzle's inner diameter and its discharge coefficient. The fluid flow from a piston pump to a cutting head is dedicated to a positive flow direction.

Besides the outtake duct, a pressure cylinder also contains an intake duct, which has a similar check-valve to restrict the fluid flow to the desired intake direction. A periodic piston displacement passively determines the switching of both check-valves as depicted in Figure 6. The resulting pumping cycle is thus divided into three states:

(a) Precharging. The piston is entirely retracted, the pumping chamber is filled with water and the pressure within the chamber is equal to the intake pressure of the water supply. While the piston extends with a velocity $w_{1}$, the 
precharge pressure within the chamber rises. Both check-valves will stay closed until the chamber's pressure reaches the actual operating pressure of the high-pressure network.

(b) Hauling. The pumping chamber is pressurized to the desired operating pressure and the piston continues to extend. Therefore, the outtake duct check-valve opens and the high-pressure network is fed with the outtake flow rate $Q_{13}$ until the piston is fully extended.

(c) Filling. The piston retracts, the chamber's pressure sinks rapidly and the outtake flow stops. This forces the outtake duct check-valve to close. As soon as the chamber's pressure drops below the supply pressure, the intake duct check-valve to the water supply will open and the chamber will be refilled with the intake flow rate $Q_{01}$.

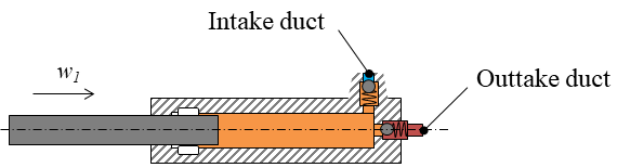

(a) Precharging
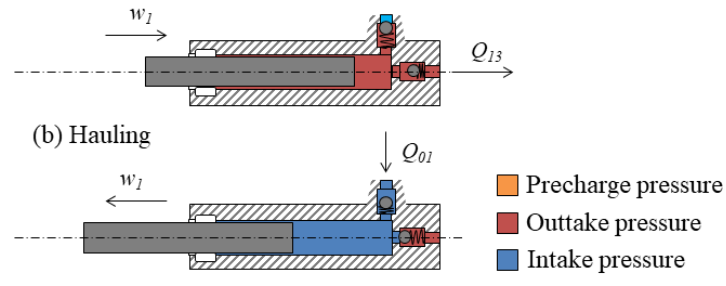

(c) Filling

Figure 6: Functional principle of a piston pump illustrated at the three states of a pumping cycle.

Providing a more detailed understanding, Niederberger et al., 2014 [19] discuss how a directly driven piston pump can induce a continuous flow rate when both pistons are synchronized to phase shifted trajectories, whereas Ferrati et al., 2015 [5] predict trajectories for the condition monitoring of a phased intensifier pump.

\subsection{System segmentation and Network assignment}

The segmentation is realized following the procedure described in Section 3. In a first step, the high-pressure components are equally divided along the fluid flow path, wherever a cross section changes. Consequently, the two check-valves, the connector along with the nozzle separate the high-pressure network into two pumping chambers, a coupling, a high-pressure pipe and a cutting head. In a second step, these initial segments can be subdivided, if needed, to represent the desired dynamics of pressure generation as induced by the piston pumps. Thus, the high-pressure pipe of length $L=4.8 \mathrm{~m}$ is further separated into $n=4$ segments of length $l=1.2 \mathrm{~m}$ to obtain a propagation time $\tau$ of less than $1 \mathrm{~ms}$.

Table 1 assigns the high-pressure network of Figure 5 to $N=8$ nodes. All nodes are interconnected as schematically illustrated in Figure 7. The resulting graph network consists of:

$$
k j \in\{[13],[23],[34],[45],[56],[67],[78],[80]\}
$$

branches and:

$$
k \in\{1,2,3,4,5,6,7,8\}
$$

nodes of homogeneous segments. By applying (32) to the assignments (33) and (34), the fluid flow path is recovered, resulting in a lumped parameter model that describes a spatially distributed high-pressure system. The number of segments $N$ defines the model's order. Each segment corresponds to a node: the pumping chambers $k=\{1,2\}$, coupling $k=3$, high-pressure pipes $k=\{4 \ldots 7\}$ and cutting head $k=8$. This yields a model of order 8 . As the final 6 segments are serially interconnected $k j=\{[34] \ldots[80]\}$, the pumping chambers follow independent flow paths $k j=\{[13],[23]\}$ that are coupled at segment $k=3$. 
Table 1: Assignment of the different high-pressure components to nodes $k$ and branches $j, k$.

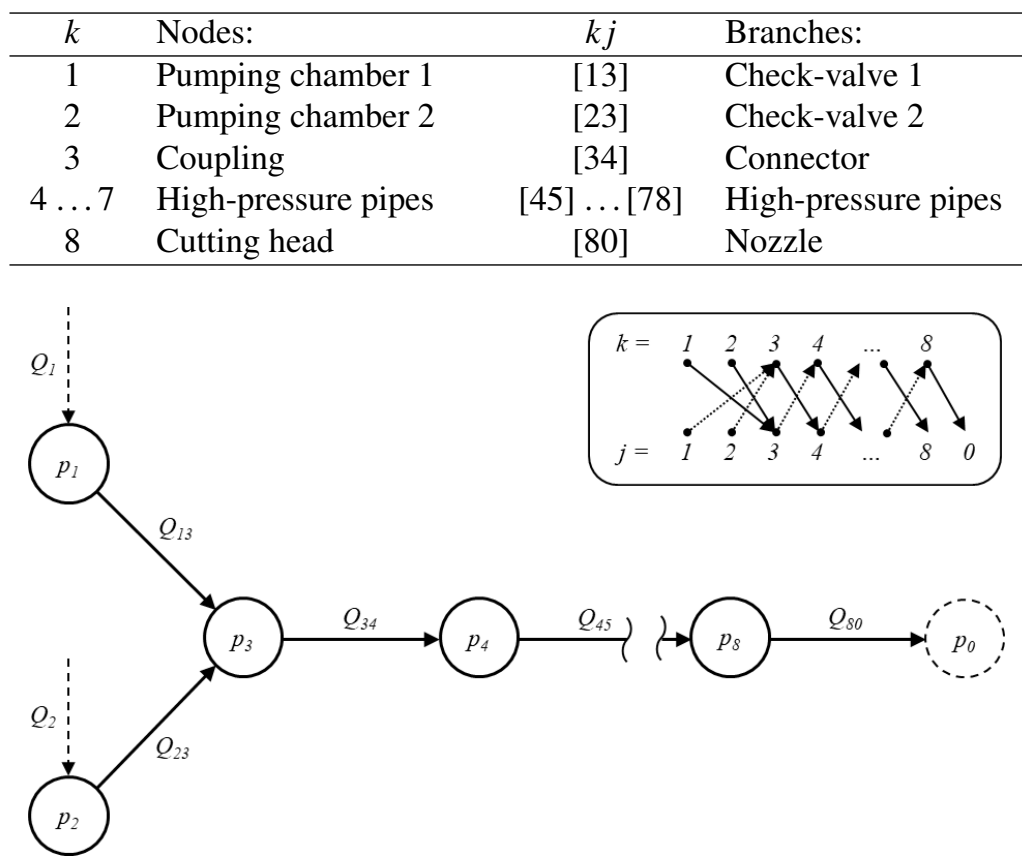

Figure 7: Scheme to assign the investigated high-pressure network to homogeneous segments of nodes and branches.

Table 2 shows the parameters of each segment. The functions $f_{13}(\cdot)$ and $f_{23}(\cdot)$ restrict the fluid flow of the checkvalves to its positive direction as each piston induces the displacement flow rates $Q_{1}$ and $Q_{2}$.

Table 2: Parameters of each segment corresponding to (33), (34) and grouped in nodes and branches.

\begin{tabular}{cccccccc}
\hline & $\begin{array}{c}S_{k} \\
\left(\mathrm{~mm}^{2}\right)\end{array}$ & $\begin{array}{c}l_{k} \\
(\mathrm{~mm})\end{array}$ & $\begin{array}{c}Q_{k} \\
\left(\mathrm{~cm}^{3} / s\right)\end{array}$ & $k j$ & $\begin{array}{c}S_{k j} \\
\left(\mathrm{~mm}^{2}\right)\end{array}$ & $\begin{array}{c}\alpha_{k j} \\
(-)\end{array}$ & $\begin{array}{c}f_{k j} \\
(-)\end{array}$ \\
\hline 1 & 229.65 & 221 & $Q_{\text {Ext. }}(t)$ & {$[13]$} & 1.77 & 0.785 & $f\left(\Delta p_{13}\right)$ \\
2 & 229.65 & 221 & $Q_{\text {Ext. }}(t)$ & {$[23]$} & 1.77 & 0.785 & $f\left(\Delta p_{23}\right)$ \\
3 & 8.04 & 790 & 0 & {$[34]$} & 4.52 & 0.351 & 1 \\
$4 \ldots 7$ & 4.52 & 1200 & 0 & {$[45] \ldots[78]$} & 4.52 & 0.193 & 1 \\
8 & 18.09 & 180 & 0 & {$[80]$} & $\{31.4,70.7\} 10^{-3}$ & 0.647 & 1 \\
\hline
\end{tabular}

The proposed modelling methodology directly allocates the parameters of high-pressure components. However, the discharge coefficient $\alpha_{k j}$ of each interconnection needs approximation by estimating the friction loss coefficient:

$$
\lambda=\frac{c_{1}}{R e^{c_{2}}}
$$

with $c_{1}=0.3164$ and $c_{2}=0.25$ for turbulent flow characteristics [24] as a Reynolds number $R e>2320$ was assumed. If the cross section $S_{k}$ changes along its fluid flow path, the discharge coefficient (21) needs to be adjusted according to [22]:

$$
\alpha_{k j}=\sqrt{\frac{1}{1+l_{k} / D_{k} \lambda-\left(1-\Delta S_{k j} / S_{k j}\right)^{2}}}
$$

whereby $\Delta S_{k j}=S_{k}-S_{k j}$ denotes the cross section difference. For example, the evaluation of (36) for a constant cross section with $l=1.2 \mathrm{~m}$ and $D=2.4 \mathrm{~mm}$ yields $\alpha>0.2$. The flow resistance further increases, when a fluid flow path 
is deflected in its direction. A discharge coefficient of $\alpha>0.7$ is typically chosen for $90^{\circ}$ angles as mentioned in [25]. In any case, the cutting heads have a dominant flow resistance because of the very small nozzle's inner diameters and their maximal pressure differences. A nozzle releases the pressure entirely, when it generates a waterjet. A discharge coefficient of $\alpha=\left[\begin{array}{lll}0.6 & 0.7\end{array}\right]$ has been obtained in experiments [2].

The derived high-pressure network $\mathcal{N}_{k j}$ from (33) and (34) has been numerically simulated in MATLAB Simulink and validated with measurements. The following conditions must be met to ensure modelling and implementation:

- A high-pressure network is divided into segments $k \in J$ of constant cross section $S_{k}$.

- All high-pressure components are considered to be ideal, e.g. the pressure stress will not cause any recognizable expansion and leakages are negligible.

- Degradation of components, such as nozzle wear, is not modelled.

- The water supply is not part of the high-pressure network. Instead, the supply pressure defines the lower bounds for all pressure states such as $p_{k}(t) \geq p_{\text {Sup. }} \forall t$.

- The initial condition $p_{k}(t=0)=p_{S \text { up }}$. requires the entire high-pressure network to be filled with water.

- Any piston displacement influences the chamber volume $V_{k}(t)$ and, thereby, the dynamics of pressure generation. The piston position is determined by a trajectory hence the volume variation is a priori known.

- As the pressure difference between each segment $\Delta p_{k j}(t)$ almost disappears for a steady state, the modelling error from discharge coefficient estimation becomes small.

- When introducing the function $f_{k j}(\cdot)$, no switching dynamics are modelled.

\section{Experimental studies}

In this section, the two model coefficients $\kappa_{0}$ and $\kappa_{1}$ of the parametric model (17) are first identified, independent of any specific test bench setup. This parametric model is required to describe the pressure-dependent bulk modulus, which is a fluid characteristic parameter that is ideally unaffected by high-pressure components or network topologies. Afterwards, the above modelled high-pressure network $\mathcal{N}_{k j}$ is validated via experimental data. A pretest analysed expected pressure overshoots and losses for an operating pressure around $350 \mathrm{MPa}$. The test bench was thereby equipped with a nozzle which has an inner diameter of $0.3 \mathrm{~mm}$. A subsequent test varied the piston's displacement flow rates to obtain an operating pressure between 100 and $400 \mathrm{MPa}$ for a $0.2 \mathrm{~mm}$ nozzle.

\subsection{Parameter identification}

The bulk modulus of water is often regarded as a constant $K_{0}=2.08 \mathrm{GPa}$. This should only be used for relatively small changes of pressure close to $10 \mathrm{MPa}$. However, the bulk modulus increases to $2.68 \mathrm{GPa}$ for water pressurized to $100 \mathrm{MPa}$. Detailed studies have analysed fluid density as well as bulk modulus [26] and tabular data has been collected for a pressure range of 0.1 to $100 \mathrm{MPa}$ [27]. This tabular data contains information to estimate a pressure-dependent bulk modulus, but further experimental data is necessary to validate the parametric model (17) for a pressure range up to $400 \mathrm{MPa}$.

Similar to the relation (18), the bulk modulus can also be expressed as [20], [22]:

$$
K(p)=-\frac{d p}{d V / V}
$$

This equation defines that any variation in fluid volume $V$ induces a change in pressure $p$. As a consequence, a piston displacement pressurizes the water within a pumping chamber. If a pumping chamber is entirely shot, the measured piston position determines the volume variation according to (13). The pressure increase is then captured by a high-pressure sensor. Data acquisition is performed at a sampling rate of $10 \mathrm{~Hz}$. 
Table 3 illustrates the design of experiment. The initial pumping chamber volume is varied for each run by choosing a different initial piston position, influencing the sensitivity of pressure generation. When performing this experiment, the piston is slowly displaced so that a nearly isothermal process of pressure generation is expected while the fluid temperature remains constant. The various runs are assigned to two different pumping chambers to eliminate volume uncertainties. In addition, all runs are repeated for two different velocities. Evidence for adiabatic effects was not found.

Table 3: Design of experiment for parameter identification to determine the pressure-dependant bulk modulus.

\begin{tabular}{cccc}
\hline Run & Pumping chamber & $\begin{array}{c}\text { Initial position } \\
(\mathrm{mm})\end{array}$ & $\begin{array}{c}\text { Piston velocity } \\
(\mathrm{mm} / \mathrm{s})\end{array}$ \\
\hline $1 \ldots 8$ & 1 & $\{0,40,80,120\}$ & $\{1,2\}$ \\
$9 \ldots 16$ & 2 & $\{20,60,100,140\}$ & $\{1,2\}$ \\
\hline
\end{tabular}

A numerical approximation of (37) with central differences yields:

$$
K\left(p_{i}\right)=-\frac{p_{i+1}-p_{i-1}}{V_{i+1}-V_{i-1}} V_{i}
$$

which evaluates each measured sample $i$ relative to its actual volume. Figure 8 (a) shows the estimated bulk modulus of all runs with respect to its pressure state. A drift is observed in the measured data of higher and lower pressure due to acceleration and deceleration of the piston. Therefore, a robust least-square method is applied to fit a 1st order polynomial. The least absolute residual method [28] was examined to generate a linear regression model with tight confidence intervals. Table 4 presents the identified coefficients. The resulting parametric model fits the data with a determination coefficient of $r^{2}=0.9959$. The prediction errors are reasonable with a sum of square error of $s s e=4.0759$ and a root mean square error of rmse $=0.0382$. The experimental data is further compared to tabular data [27], see Figure 8 (b), whereby a offset is observed. This may be caused due to a static pressure head of the test bench.
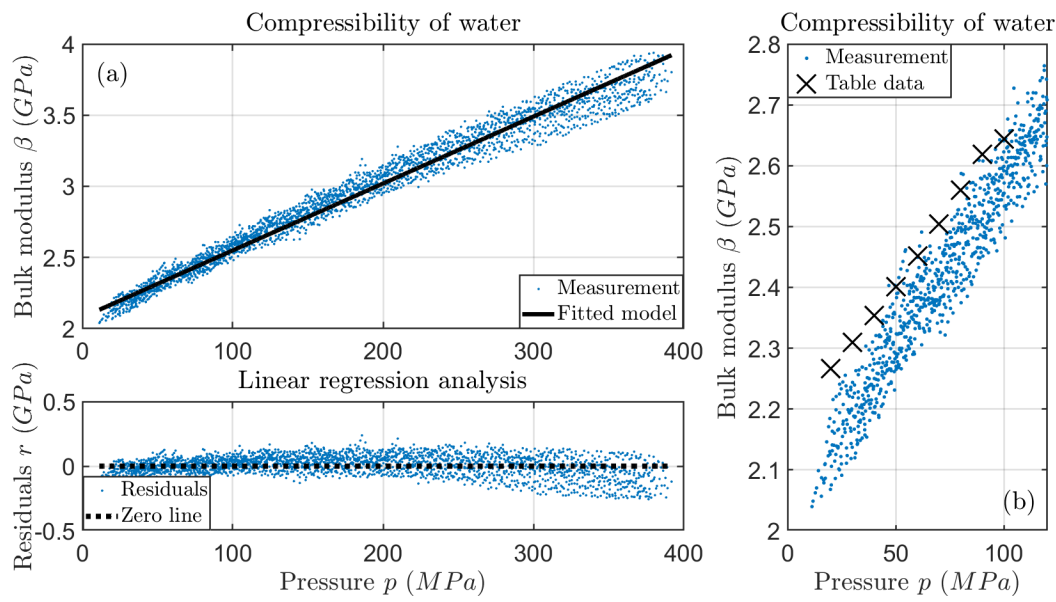

Figure 8: Pressure-dependent bulk modulus has been derived from experimental data for model fitting (a) and compared to tabular data (b).

Table 4: Identified coefficients of the parametric model and its confidence intervals describing the pressure-dependent bulk modulus.

\begin{tabular}{cccc}
\hline Coefficient & Fitted value & $95 \%$ confidence bounds & Unit \\
\hline$\kappa_{0}$ & 2.079 & {$[2.077,2.081]$} & $(\mathrm{GPa})$ \\
$\kappa_{1}$ & 4.703 & {$[4.693,4.712]$} & $(-)$ \\
\hline
\end{tabular}




\subsection{Model validation}

The proposed graph-based modelling methodology was verified on the introduced supply network for waterjet machining. Figure 9 illustrates the experimental model validation. For each experiment, reference position trajectories were generated and loaded to the test bench PLC. Pistons were periodically displaced according to these reference trajectories. The set point variable $r_{s}$ defines the cycle time of piston displacement to obtain the desired fluid flow rate. The actual piston positions, the velocities $u$ as well as the pressure trends $y$ were measured at a sampling rate of $200 \mathrm{~Hz}$. Data acquisition of $u_{m}$ and $y_{m}$ was realized using the hardware mentioned in Table 5. The measured position and velocity data $u_{m}$ was then directly fed into MATLAB Simulink for numerical simulation, whereby the simulated pressure trends $y_{e}$ were compared to measurements $y_{m}$.

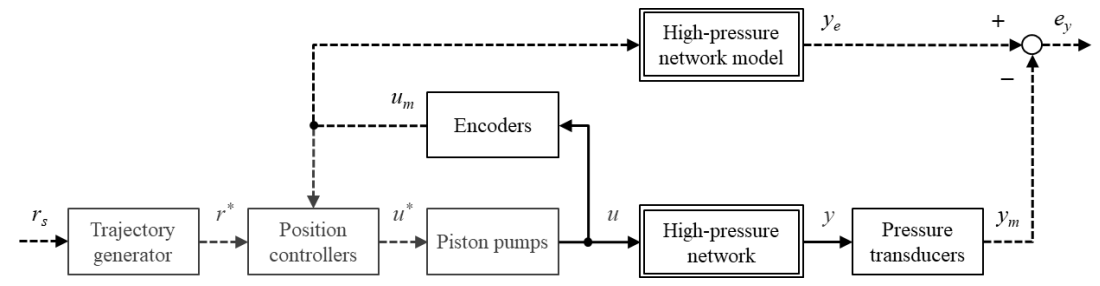

Figure 9: Block diagram providing an overview of the model validation. The measured piston positions and velocities are used as the model inputs. The simulated model output has been compared to the measured pressure.

Table 5: Hardware configuration for data acquisition.

\begin{tabular}{llll}
\hline & Name & Type & Properties \\
\hline$y_{m}$ & Pressure transducers: & 2x Gefran, & $0.1 \%$ accuracy, \\
& & TPHADA-N-E-V-B05M-T. & $<1$ ms response time. \\
& \multirow{2}{*}{ Analog input module: } & 1x Rockwell, & $0.2 \%$ accuracy, 16 bit resolution, \\
& & Compact I/O 5069-IY4. & $\geq 0.7$ ms sampling rate. \\
$u_{m}$ & Absolute encoders: & 2x Sick, & 32 '768 steps/revolution, \\
& & SRM50-FFA0-S21. & 12 bit resolution. \\
& \multirow{2}{*}{ Servo drives: } & 2x Rockwell, & HIPERFACE-Interface, \\
& & Kinetix 5700 2198-S160-ERS3. & $\geq 0.2$ ms packet interval. \\
\hline
\end{tabular}

A first measurement series, the pretest, analyses the excitation characteristics of the investigated supply network, whereas a second measurement series, the subsequent test, verifies the accuracy of the high-pressure network model.

\subsubsection{Pretest}

This first measurement series validates the passive coupling between the interconnected piston pumps using two experiments. Both experiments were conducted with the same position trajectories. These trajectories were optimized for an operating pressure of $350 \mathrm{MPa}$ which refers to the trajectory reference value. A nozzle of $0.3 \mathrm{~mm}$ inner diameter was installed to the cutting head $\left(S_{80}=70.7 \cdot 10^{-3} \mathrm{~mm}^{2}\right)$. Consequently, a displacement flow rate of $35.5 \mathrm{~cm}^{3} / \mathrm{s}$ would be ideal to generate a pressure of $350 \mathrm{MPa}$. However, the pump control values were slightly modified so that experiment 1 would clearly show the effects of over excitation, whereas experiment 2 would cause an under excitation. Both experiments will be described below.

Experiment 1 - Over excitation. The displacement flow rate was raised to $r_{380}=37.0 \mathrm{~cm}^{3} / \mathrm{s}$ to obtain an increased pressure steady state of about $380 \mathrm{MPa}$. The insufficient precharging leads to a periodic pressure loss towards $350 \mathrm{MPa}$, as can be observed in Figure 10 (a). However, the pressure will asymptotically rise towards the desired value, once the check-valve of the precharged pumping chamber has been pushed open.

Experiment 2 - Under excitation. The displacement flow rate was reduced to $r_{320}=34.5 \mathrm{~cm}^{3} / \mathrm{s}$. This results in a decreased pressure steady state of about $320 \mathrm{MPa}$. The excessive precharging causes a periodic pressure overshoot towards $350 \mathrm{MPa}$, cf Figure 10 (b). Complementary to the first experiment, the pressure will asymptotically fall towards the desired value, once the check-valve is closed. 

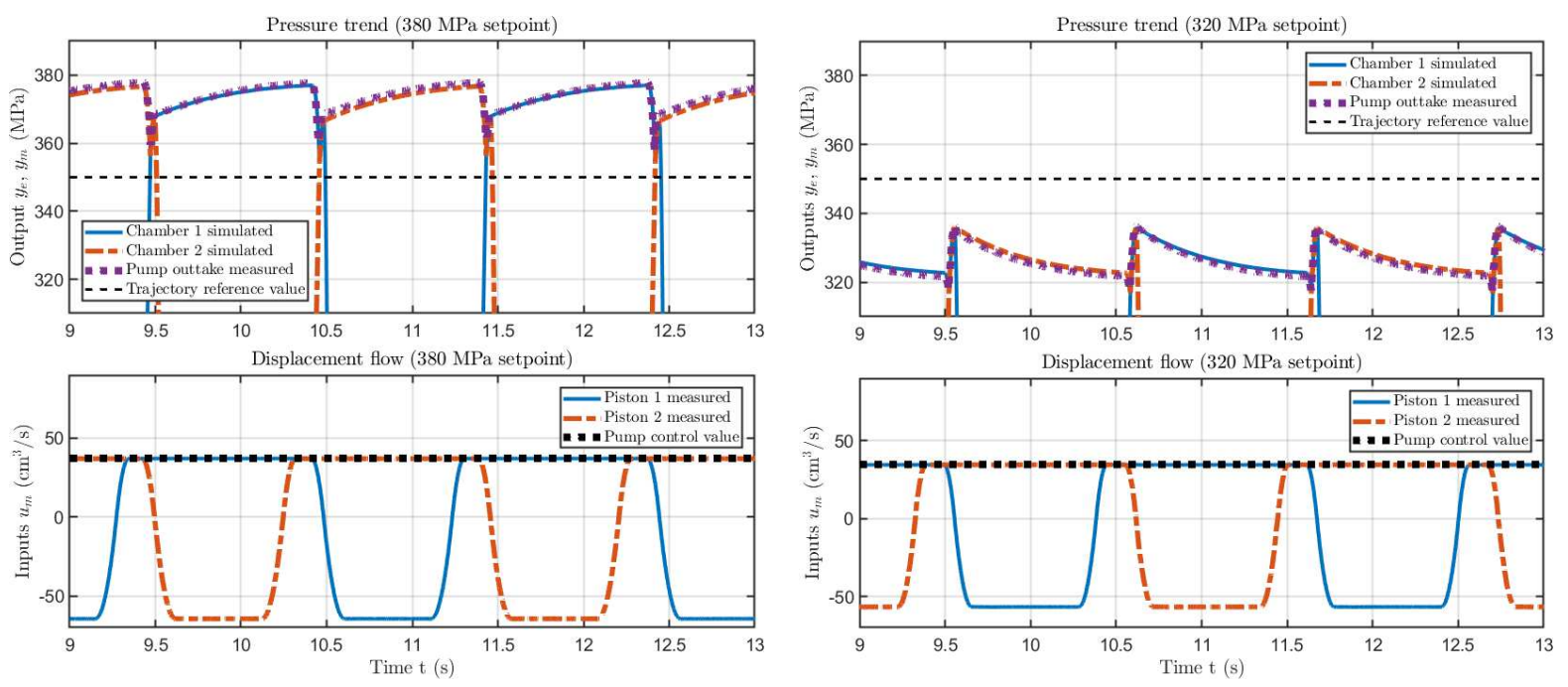

(a) Experiment 1. Over excitation for $37.0 \mathrm{~cm}^{3} / \mathrm{s}$ displacement flow causes a periodic pressure loss.

(b) Experiment 2. Under excitation for $34.5 \mathrm{~cm}^{3} / \mathrm{s}$ displacement flow cases a periodic pressure overshoot.

Figure 10: Pretest - Measured pump outtake pressure compared to simulated pressure states and corresponding displacement flow rates to excite the high-pressure network. Test bench setup using a $0.3 \mathrm{~mm}$ nozzle and a position trajectory optimized for $350 \mathrm{MPa}$.

The pressure generation is sensitive to any deviations of the ideal pump control value (desired displacement flow). It has to be noticed remarkably that the simulation reproduced the discussed effects with the desired accuracy. The pumping chambers with the pressure states $p_{1}$ and $p_{2}$ affect the total pump outtake pressure $p_{3}$. The pressure differences $\Delta p_{13}$ and $\Delta p_{23}$ over the corresponding check-valves restrict the induced fluid flows $Q_{13}$ and $Q_{23}$ to a positive direction with respect to the switching laws $f_{13}(\cdot)$ and $f_{23}(\cdot)$. Detailed pressure trends of both experiments are shown in Figures 11 (a) and (b).

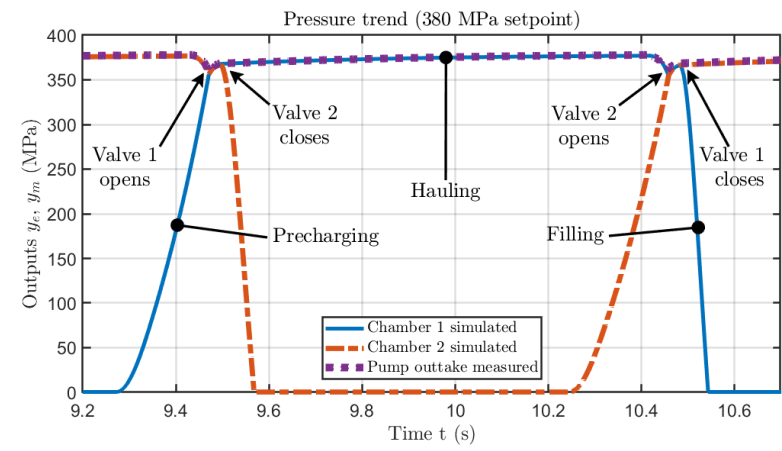

(a) Experiment 1. Enlarged plot in Figure 10 (a) showing the pressure trend at a $380 \mathrm{MPa}$ steady state.

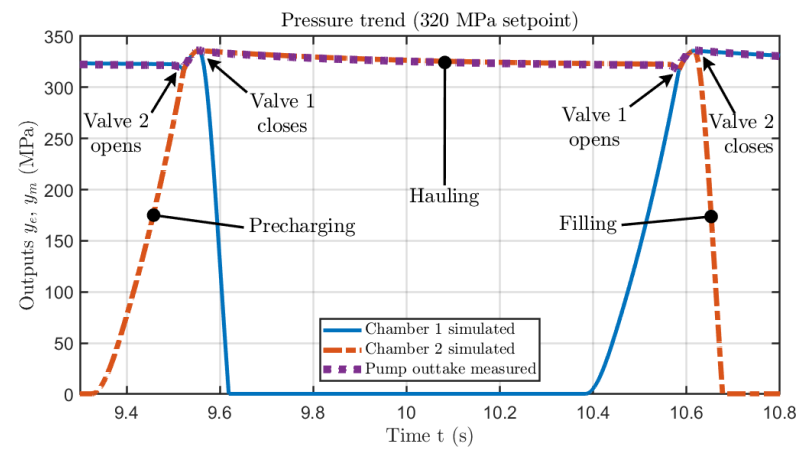

(b) Experiment 2. Enlarged plot in Figure 10 (b) showing the pressure trend at a $320 \mathrm{MPa}$ steady state.

Figure 11: Pretest - Simulated pumping chamber pressures through out a pumping cycle and resulting pump outtake pressure due to check-valve switching hysteresis.

The three states of a pumping cycle (precharging, hauling and filling) as introduced in Section 4 are clearly distinguishable. Depending on the amount of precharging, pressure losses or pressure overshoots can occur when a check-valves opens too early or, respectively too late. The check-valves are passively controlled by any piston displacement that pressurizes the corresponding pumping chamber. Consequently, a piston displacement indirectly affects the coupling between segments 1, 2 and 3. Trieb et al., 2007 [29] have observed similar behaviours for the pressure generation of intensifier pumps. 


\subsubsection{Subsequent test}

This second measurement series analyses how the proposed model performed with an operating range of 100 to $400 \mathrm{MPa}$. The measurement shown in Figure 12 resulted from position trajectories optimized for $200 \mathrm{MPa}$ pressure. Operating points below optimal pressure cause increasing pressure overshoots and operating points above an optimal pressure result in increasing pressure losses. In contrast to the pretest, a nozzle with an inner diameter of $0.2 \mathrm{~mm}$ was used $\left(S_{80}=31.4 \cdot 10^{-3} \mathrm{~mm}^{2}\right)$. This notably influences the system's characteristics. Therefore, a displacement flow rate of $r_{200}=19.0 \mathrm{~cm}^{3} / \mathrm{s}$ was needed to obtain a steady pressure trend of $200 \mathrm{MPa}$. During the measuring procedure, the reference displacement flow rate was varied to follow a stair function according to:

$$
r_{s}=\{0.6,0.8,1.0,1.2,1.4,1.2,1.0,0.8,0.6\} \cdot r_{200}
$$

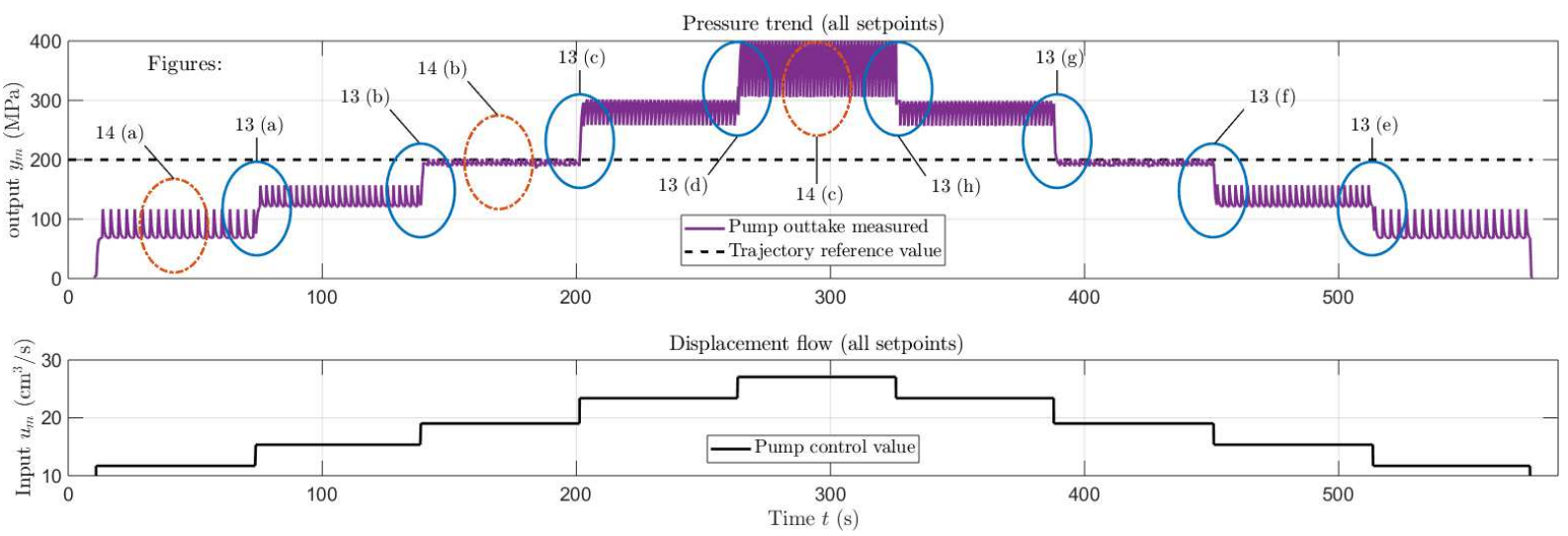

Figure 12: Subsequent test - Measured pump outtake pressure and corresponding reference displacement flow rate. High-pressure network configured with a $0.2 \mathrm{~mm}$ nozzle and position trajectory optimized for $200 \mathrm{MPa}$.

The simulation results were obtained with Simulink $22017 b$ using the ode $23 s$ solver with variable step size. The solver was configured for a relative tolerance of $10^{-4}$ and an absolute tolerance of $10^{-3}$. It was running on a Windows 10 machine with an Intel Core i7-7600U $2.80 \mathrm{GHz}$ processor and 16.0 GB RAM. For this particular case, an average simulation time of 63.84 seconds can be reported which corresponds to a real-time factor of 8.87 .

Figure 13 illustrates the measured and simulated pressure trends for each step as applied to the displacement flow rate. The simulated transient behaviour as well as the resulting operating points are promising. Figure 14 compares the measured with the simulated pressure trends for the operating points at 100, 200 and $400 \mathrm{MPa}$ as well as the resulting residuals are shown. Additional to the proposed modelling methodology, a model for constant bulk modulus with $K_{0}=2.7374 \mathrm{GPa}$ and a model for constant fluid density with $\rho_{0}=1104.6 \mathrm{~kg} / \mathrm{m}^{3}$ was considered. The constant parameters were individually obtained for each model to minimize the resulting root mean square error $r m s e$ between the simulated pump outtake pressure and the experimental data. The constant bulk modulus of minimal rmse was found for a pressure of $140 \mathrm{MPa}$ and the constant fluid density of minimal rmse was found for a pressure of $270 \mathrm{MPa}$. In contrast, the proposed variable parameter model requires no specific tuning for the investigated high-pressure network.

It has been recognized that a pressure-dependent bulk modulus is essential to track the transient behaviour over the desired operating range. Applying a constant bulk modulus results in an incorrect transient behaviour that converges towards the desired steady state. In contrast, a constant fluid density causes a transient behaviour similar to the measurement but with an incorrect steady state. Therefore, a pressure-dependent fluid density is desired to meet the steady state over a wide operating range. Table 6 lists the correlation coefficient $R$ and the root mean square error $r m s e$ for all the discussed models. The evaluation is based on the experimental and simulation data over a time interval of 30 seconds at a constant operating point. All models achieved a correlation coefficient of $R>0.9377$. As the proposed modelling methodology obtained a root mean square error of $r m s e<1.62$ for all observed operating points, an increase to rmse $>8.82$ was observed for both models of constant parameter. Other modelling methodologies 

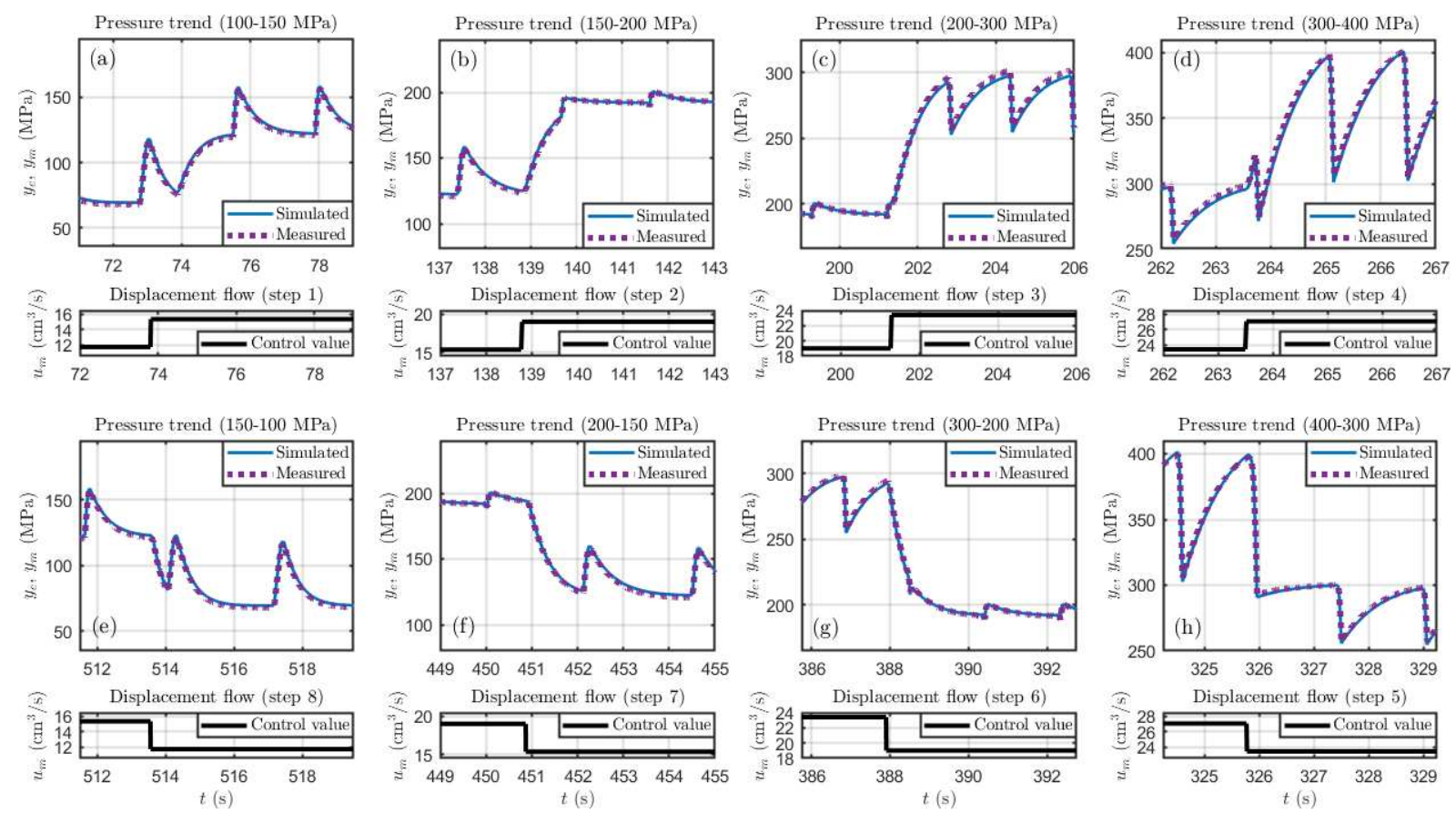

Figure 13: Subsequent test - Extract of measured and simulated pump outtake pressure for each reference step.
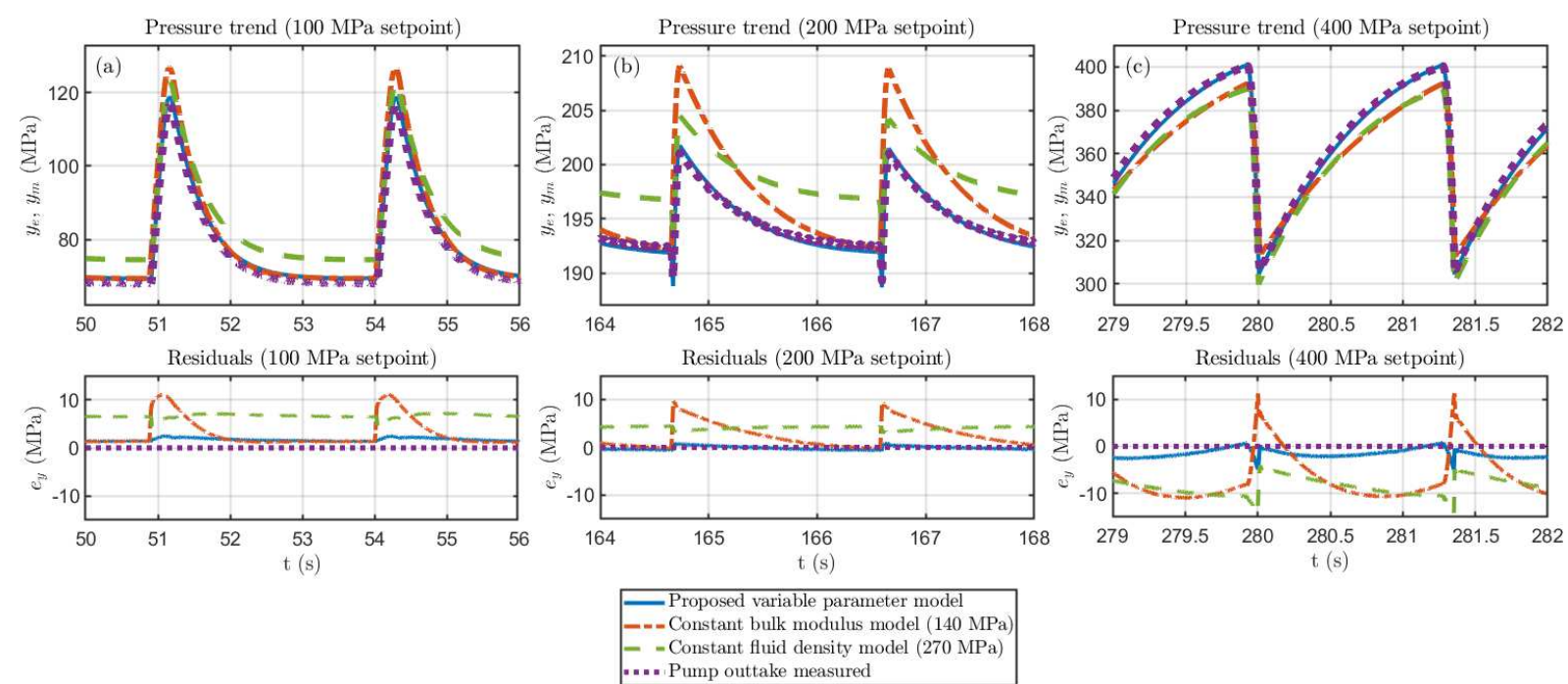

Figure 14: Subsequent test - Extract of measured and simulated pump outtake pressure for different operating points. The proposed modelling methodology is thereby compared to simulations with constant bulk modulus and constant fluid density.

which only partially consider variable fluid densities ([4], [5]) or variable bulk modulus ([7], [9]) are sufficient for simulations around a specific operating point. However, the proposed methodology improves the simulation's accuracy when a wide pressure range is desired.

The enlarged plots in Figure 15 show the experimental and simulated data around transient peaks. These details originate from the plots in Figure 14. In general, the experimental data indicates reasonable noise dispersion in relation to the observed dynamics and amplitudes of pressure generation. 
Table 6: Correlation coefficients and root mean square errors to compare the model's accuracy for different operating pressures.

\begin{tabular}{lccccccc}
\hline & \multicolumn{2}{c}{$100 \mathrm{MPa}$} & \multicolumn{2}{c}{$200 \mathrm{MPa}$} & \multicolumn{2}{c}{$400 \mathrm{MPa}$} & \multicolumn{2}{c}{ overall } \\
Modelling methodology: & $R$ & rmse & $R$ & rmse & $R$ & rmse & rmse \\
\hline Proposed variable parameters & 0.9998 & 1.62 & 0.9956 & 1.37 & 0.9991 & 1.45 & 2.12 \\
Constant bulk modulus $\left(K_{0}=2.7374 \mathrm{GPa}\right)$ & 0.9927 & 4.07 & 0.9377 & 3.64 & 0.9883 & 8.82 & 6.03 \\
Constant fluid density $\left(\rho_{0}=1104.6 \mathrm{~kg} / \mathrm{m}^{3}\right)$ & 0.9989 & 6.53 & 0.9955 & 4.11 & 0.9982 & 9.40 & 7.25 \\
\hline
\end{tabular}
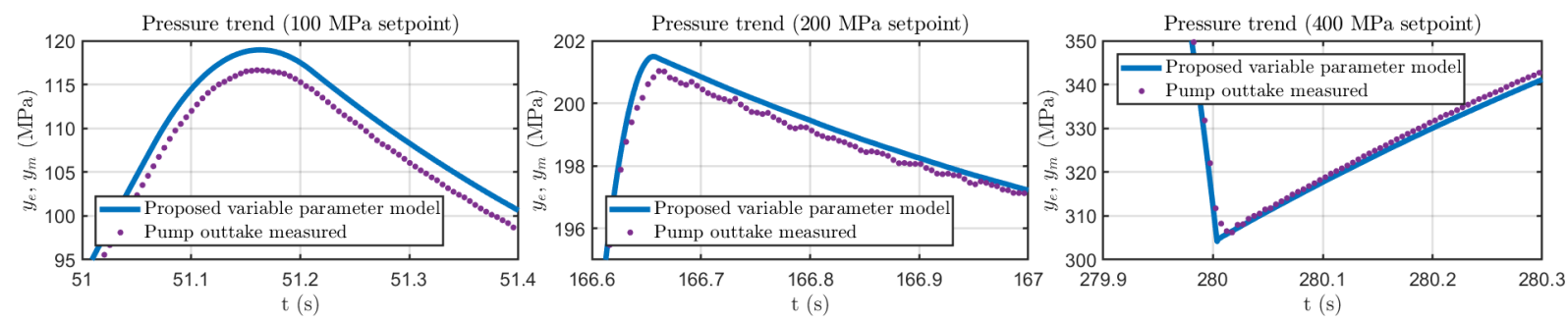

Figure 15: Subsequent test - Zoomed in around transient peaks of measured and simulated pump outtake pressure for different operating points.

\section{Discussion and Conclusion}

In this paper, a graph-based methodology has been proposed to model high-pressure generation in a supply network for waterjet machining. This modelling methodology assigns the components of a high-pressure system to homogeneous segments. These segments are then interconnected by means of graphs to recover the initial network topology. Each segment is represented as a local pressure state and its interconnections are characterized by a onedimensional stationary fluid flow. The result is a lumped parameter model that can be easily implemented in MATLAB Simulink for numerical simulations.

Further, a variable fluid compressibility was taken into account when a variable fluid volume and a pressuredependent bulk modulus was introduced. Any displacement flow was considered to be an external excitation and a piston displacement was modelled by a change in the fluid volume. The bulk modulus, however, was represented by a parametric model that was further used to derive a pressure-dependent fluid density. Its coefficients were experimentally identified when a determination coefficient of $r^{2}=0.9959$ was achieved.

The derived model of the investigated supply network for waterjet machining was validated with experimental data using a test bench. It was found that modelling a constant fluid density caused a steady state pressure error and a constant bulk modulus was responsible for divergent transient behaviour. Therefore, an improved model accuracy was achieved for a desired operating range of 100 to $400 \mathrm{MPa}$ and a robust pressure prediction was obtained as the overall rmse remained below $2.12 \mathrm{MPa}$ when a variable fluid compressibility was considered.

It was further recognized that the modelled switching behaviour is very sensitive to any pressure mismatches. An accurate switching hysteresis, therefore, will reduce the resulting simulation residuals. Pressure losses or overshoots were observed as the applied trajectories imperfectly synchronized the pistons. The derived model reproduced this behaviour and can be used to improve the pressure generation by means of optimized position trajectories.

Using the proposed methodology, it is possible to flexibly model various network topologies. As the test bench is capable of representing even more complex supply networks with up to four piston pumps and two cutting heads, detailed studies are planned to test the full ability of the modelling methodology. This graph-based methodology eventually intends to model an entire class of distributed high-pressure networks. The modelling is essential to optimize complex supply networks for waterjet machining and decentralized pressure control techniques will be further investigated, but without the need to individually validate each network model with work intensive measurements. Further work should identify the presently estimated model parameters of high-pressure components and continue to validate the proposed modelling methodology on more complex high-pressure networks. 


\section{Acknowledgements}

The authors would like to acknowledge the research collaboration with Jet Cut Power, Rotkreuz and Zaugg Maschinenbau, Schönenwerd as well as the financial support provided by the Swiss Commission for Technology and Innovation CTI. The authors are grateful to Jean-Paul John and Pascal Zeugin for their efforts to upgrade the test bench and for their support while running the experiments.

\section{References}

[1] J. Folkes, Waterjetan innovative tool for manufacturing, Journal of Materials Processing Technology 209 (20) (2009) 6181 - 6189, special Issue: 1st International Conference on Abrasive Processes.

[2] T. Susuzlu, A. Hoogstrate, B. Karpuschewski, Initial research on the ultra-high pressure waterjet up to 700mpa, Journal of Materials Processing Technology 149 (1) (2004) 30 - 36, 14th Interntaional Symposium on Electromachining (ISEM XIV).

[3] M. Tremblay, M. Ramulu, Modeling and simulation of pressure fluctuations in high pressure waterjets, in: 10th American Waterjet Conference, no. 12, WaterJet Technology Association WJTA, 1999.

[4] B. C. Fabien, M. Ramulu, M. Tremblay, Dynamic modelling and identification of a waterjet cutting system, Mathematical and Computer Modelling of Dynamical Systems March 2003 (2010) 45-63.

[5] G. Ferretti, M. Monno, B. Scaglioni, M. Goletti, M. Grasso, Abrasive waterjet intensifier model for machine diagnostics, in: Proceedings of the 11th International Modelica Conference, 2015.

[6] A. W. Momber, A generalized abrasive water jet cutting model, in: 8th American Water Jet Conference, no. 25, Water Jet Technology Association WJTA, 1995.

[7] D. Hountalas, A. Kouremenos, Development of a fast and simple simulation model for the fuel injection system of diesel engines, Advances in Engineering Software 29 (1) (1998) 13 - 28.

[8] M. Wang, G. Ouyang, J. Zhou, Simulation and experimental study on high pressure common rail fuel system of diesel engine, in: 2011 International Conference on Computer Distributed Control and Intelligent Environmental Monitoring, (IEEE), 2011.

[9] P. Lino, B. Maione, A. Rizzo, Nonlinear modelling and control of a common rail injection system for diesel engines, Applied Mathematical Modelling 31 (9) (2007) 1770 - 1784

[10] H. Wang, D. Zheng, Y. Tian, High pressure common rail injection system modeling and control, ISA Transactions 63 (2016) 265 - 273.

[11] L. Ford, D. Fulkerson, Flows in Networks, Princeton Legacy Library, Princeton University Press, 2016.

[12] N. Espitia, A. Girard, N. Marchand, C. Prieur, Fluid-flow modeling and stability analysis of communication networks, IFAC-PapersOnLine 50 (1) (2017) 4534 - 4539, 20th IFAC World Congress.

[13] A. van der Schaft, Modeling of physical network systems, Systems \& Control Letters 101 (Supplement C) (2017) 21 - 27, jan C. Willems Memorial Issue, Volume 2.

[14] J. P. Koeln, M. A. Williams, H. C. Pangborn, A. G. Alleyne, Experimental validation of graph-based modeling for thermal fluid power flow systems, in: Proceedings of the ASME 2016 Dynamic Systems and Control Conference, 2016.

[15] H. C. Pangborn, M. A. Williams, J. P. Koeln, A. G. Alleyne, Graph-based hierarchical control of thermal-fluid power flow systems, in: 2017 American Control Conference (ACC), IEEE, 2017.

[16] T. F. Corbet, W. Beyeler, M. L. Wilson, T. P. Flanagan, A model for simulating adaptive, dynamic flows on networks: Application to petroleum infrastructure, Reliability Engineering \& System Safety 169 (Supplement C) (2018) 451 - 465.

[17] R. Borsche, J. Kall, High order numerical methods for networks of hyperbolic conservation laws coupled with odes and lumped parameter models, Journal of Computational Physics 327 (Supplement C) (2016) 678 - 699.

[18] A. P. Ramallo-Gonzlez, M. E. Eames, D. A. Coley, Lumped parameter models for building thermal modelling: An analytic approach to simplifying complex multi-layered constructions, Energy and Buildings 60 (Supplement C) (2013) $174-184$

[19] S. Niederberger, L. Kurmann, Energy-efficient high-pressure pump, in: Proceedings of the 14th Mechatronics Forum International Conference, Mechatronics 2014, Karlstad University, Sweden, 2014, pp. 132-140.

[20] V. L. Streeter, E. Wylie, K. Bedford, Fluid Mechanics, Civil engineering series, WCB/McGraw Hill, 1998.

[21] V. K. Gupta, Z. Zhang, Z. Sun, Modeling and control of a novel pressure regulation mechanism for common rail fuel injection systems, Applied Mathematical Modelling 35 (7) (2011) 3473 - 3483.

[22] J. Spurk, Strömungslehre: Einführung in die Theorie der Strömungen, Springer-Lehrbuch, Springer Berlin Heidelberg, 2013.

[23] Q. Liu, X. Gong, Y. Hu, H. Chen, Active disturbance rejection control of common rail pressure for gasoline direct injection engine, in: 2013 American Control Conference, Institute of Electrical and Electronics Engineers (IEEE), 2013.

[24] H. Blasius, Das Ähnlichkeitsgesetz bei Reibungsvorgängen in Flüssigkeiten: über den Gültigkeitsbereich der beiden Ähnlichkeitsgesetze in der Hydraulik, Mitteilungen über Forschungsarbeiten auf dem Gebiete des Ingenieurwesens, VDI-Verlag, 1913.

[25] D. Will, N. Gebhardt (Eds.), Hydraulik, Springer Berlin Heidelberg, 2014.

[26] C.-T. A. Chen, F. J. Millero, Thermodynamic properties for natural waters covering only the limnological range 1, Limnology and Oceanography 31 (3) (1986) 657-662.

[27] E. Schmidt, U. Grigull, Properties of water and steam in SI-units:, Springer, 1981

[28] F. H. Thanoon, Robust regression by least absolute deviations method, International Journal of Statistics and Applications 5 (3) (2015) $109-112$.

[29] R. M. F. Trieb, R. Karl, Pressure and flow rate fluctuations at high pressure intensifier pumps, in: 2007 American WJTA Conference and Expo, Water Jet Technology Association WJTA, 2007. 